\title{
Polymorphisms in chromosome region 12q13 and their influence on age at onset of type 1 diabetes
}

\author{
L. Espino-Paisan • H. de la Calle • \\ M. Fernández-Arquero • M. Á. Figueredo • \\ E. G. de la Concha • E. Urcelay • J. L. Santiago
}

Received: 10 September 2010 / Accepted: 13 April 2011 / Published online: 11 May 2011

(C) Springer-Verlag 2011

\begin{abstract}
Aims/hypothesis A complex region covering numerous genes in 12q13 was first associated with type 1 diabetes in the Wellcome Trust Case-Control Consortium (WTCCC) study. Two studies performed in a white population have tested the association of polymorphisms within this region with age at onset of the disease, with seemingly contradictory results. We aimed at replicating three of the strongest signals in a group of patients with early and late disease onset.

Methods Polymorphisms rs773107, rs2292239 and rs10876864 were genotyped in 444 type 1 diabetic Spanish participants (age at onset $0-65$ years) and 861 controls. The influence of single nucleotide polymorphisms (SNPs) on age at onset was tested through stratified and continuous analyses.

Results rs773107 and rs2292239 were significantly associated with the disease, while rs10876864 showed a trend towards statistical significance in the whole population analyses. Comparison of early-onset patients to controls was significant for the three polymorphisms (allelic $p<0.006$ ). Late-onset patients and controls did not reveal statistical differences. Analysis of age at onset in both rs 773107
\end{abstract}

Electronic supplementary material The online version of this article (doi:10.1007/s00125-011-2177-6) contains supplementary material, which is available to authorized users.

L. Espino-Paisan $(\bowtie) \cdot$ M. Fernández-Arquero $\cdot$

M. Á. Figueredo - E. G. de la Concha • E. Urcelay • J. L. Santiago Immunology Department, Hospital Clínico San Carlos, Instituto de Investigacíon Sanitaria San Carlos (IdISSC),

Martín Lagos s/n,

28040 Madrid, Spain

e-mail: lauraep80@yahoo.es

H. de la Calle

Endocrinology Department, Hospital Ramón y Cajal,

Madrid, Spain and rs2292239 showed differences between genotypes $(p \leq 0.002)$, alleles $(p \leq 0.013)$ and homozygotes for the risk genotype $\left(p \leq 4 \times 10^{-4}\right)$. Polymorphism rs10876864 showed trends towards statistical significance in the allelic frequencies $(p=0.051)$ and homozygotes for the risk genotype $(p=0.056)$. Subjects with risk genotypes had a disease onset between 2 and 5 years earlier than carriers of protective alleles.

Conclusions/interpretation We replicate two of the previously studied associations in a Spanish population and find new evidence of the influence of the 12q13 region on age at onset of type 1 diabetes.

Keywords 12q13 - Age at onset - ERBB3 - Susceptibility . Type 1 diabetes
Abbreviations
EuroAIMS European Ancestry Informative Markers
SNP Single nucleotide polymorphism
WTCCC Wellcome Trust Case-Control Consortium

\section{Introduction}

Chromosome region 12q13 was first associated with type 1 diabetes in the Wellcome Trust Case-Control Consortium (WTCCC) genome-wide study [1]. The strongest signal, rs2292239 (ERBB3), pointed to a region of strong linkage disequilibrium that included several genes. Todd et al. [2] replicated the WTCCC signal and analysed another one, rs11171739 (5' of ERBB3, $r^{2}=0.71$ with rs2292239), finding that rs 2292239 remained the strongest marker. Both studies were performed in populations with age at disease onset under 17 years. 
A further study by Hakonarson et al. on 12q13 [3] included patients with age at diagnosis under 45 years. The authors aimed at replicating 24 signals identified in a previous genome-wide study [4] that were moderately associated with type 1 diabetes, and found the top hit associations in 12q13: rs773107 (RAB5B), rs10876864 (SUOX) and rs17017704 (IKZF4). They also conducted an age-at-onset analysis and found suggestive evidence of an effect on the age at disease onset.

In the most recent study [5], the authors genotyped 19 single nucleotide polymorphisms (SNPs), including the five previously described, in patients with paediatric and adult onset type 1 diabetes. They also performed mRNA and protein expression analysis, providing some interesting evidence of $E R B B 3$ as one of the causal genes in the region. However, they did not find evidence of age-at-onset effects in $12 \mathrm{q} 13$.

We aimed at replicating the original WTCCC signal and at evaluating the age-at-onset effect previously observed in a population with paediatric and adult onset of type 1 diabetes. For this purpose, we selected the original WTCCC signal (rs2292239) and two SNPs from the Hakonarson et al. study [3]: the one most associated with age at onset (rs773107) and the one that had lower $r^{2}$ with the original 12q13 signal (rs10876864).

\section{Methods}

Participants Spanish participants with type 1 diabetes ( $n=444,47 \%$ female) were recruited, all insulin-dependent and diagnosed according to the criteria of the ADA. Healthy controls ( $n=861,53 \%$ female) were recruited among blood donors in the Madrid area. Both controls and patients were of Europid ancestry. Control participants had previously passed genome-wide quality tests for population stratification $[6,7]$. Moreover, analysis of five EuroAIMS (European Ancestry Informative Markers) did not show evidence of population stratification either ( $p>0.15$, Electronic supplementary material [ESM] Note 1 and ESM Table 1).

Age at onset was available for 409 patients, ranging from 0 to 65 years (mean, 18.6 \pm 11.1 years; median, 16 years; interquartile range 11-25 years). Adult-onset patients were classified as having type 1 diabetes by positive autoantibodies (when available), lean body type and dependence on exogenous insulin since diagnosis. All patients with age at diagnosis over 35 years were autoantibody positive.

For stratified analyses, patients were classified as earlyonset ( $\leq 16$ years) and late-onset ( $>16$ years). This cut-off is extensively used in genome-wide association studies to exclude late-onset patients, and it is similar to the cut-off chosen in one of the previous studies on this locus [5].
Informed consent was obtained from the participants included and the study was approved by the Ethics Committee of the Hospital Clínico San Carlos.

Genotyping Patients were genotyped using TaqMan Assays C $\quad-8340401 \quad 10$ (rs773107), C $15967467 \quad 10$ (rs2292239) and $\mathrm{C}-152341110$ (rs10876864) in a $7900 \mathrm{HT}$ Fast RealTime PCR System (Applied Biosystems, Foster City, CA, USA) following manufacturer's conditions. The call rate for each SNP was over $95 \%$.

Statistical analysis No statistically significant deviations from Hardy-Weinberg equilibrium were observed. Differences in allele, genotype and carrier frequencies were calculated by $\chi^{2}$ test. Associations were estimated using ORs with 95\% CIs. Power calculation and statistical analysis was carried out using Epi Info v. 5 (CDC, Atlanta, GA, USA). Analysis of age-at-onset as a continuous variable was performed through Mann-Whitney $U$ and Kruskall-Wallis tests using SPSS v. 15.0 software (Chicago, IL, USA).

\section{Results}

We found susceptibility effects of the minor alleles of polymorphisms rs773107 and rs2292239 in the case-control analysis (Table 1). The $\chi^{2}$ test for age-at-onset stratified analysis in rs773107 revealed a significant difference in homozygotes for the risk allele, and a trend for statistical significance in homozygotes for the risk allele at rs2292239. Comparison of paediatric and adult patients with controls in both polymorphisms showed that only paediatric groups were significantly different (Table 1 , footnotes a and b). For the analysis of age as a continuous variable (Table 2), we followed the approach chosen by Hakonarson et al. [3]. Results in both polymorphisms were concordant with the associations detected in the stratified analysis, revealing that patients homozygous for the risk allele had an onset between 4 and 5 years earlier than those carriers of the protective alleles.

The case-control analysis of rs 10876864 showed a trend towards statistical significance in the allelic comparison. A trend was also seen in the age-at-onset stratified analysis (Table 1). When comparing each set of patients with controls, the paediatric group was the one significantly different (Table 1, footnote c). Analysis of age at onset as a continuous variable was concordant with the results observed in the stratified analysis and showed that homozygotes for the risk genotype progressed to disease 2 years earlier than carriers of the protective allele (Table 2). 
Table 1 Genotypic and allelic frequencies of rs773107, rs2292239 and rs10876864

\begin{tabular}{|c|c|c|c|c|c|c|c|c|c|c|c|}
\hline \multirow[t]{2}{*}{ Polymorphism } & \multicolumn{2}{|l|}{ T1D } & \multicolumn{2}{|c|}{ Controls } & \multirow[t]{2}{*}{$p$ value } & \multirow[t]{2}{*}{ OR $(95 \% \mathrm{CI})$} & \multicolumn{2}{|c|}{$\mathrm{T} 1 \mathrm{D} \leq 16$ years } & \multicolumn{2}{|c|}{$\mathrm{T} 1 \mathrm{D}>16$ years } & \multirow[t]{2}{*}{$p$ value } \\
\hline & $n$ & $\%$ & $n$ & $\%$ & & & $n$ & $\%$ & $n$ & $\%$ & \\
\hline \multicolumn{12}{|l|}{$\operatorname{rs} 773107^{\mathrm{a}}$} \\
\hline & 433 & & 832 & & & & 202 & & 199 & & \\
\hline AA & 132 & 30.5 & 314 & 37.7 & & 1 & 54 & 26.7 & 61 & 30.7 & 1 \\
\hline $\mathrm{AG}$ & 223 & 51.5 & 399 & 48.0 & 0.03 & $1.33(1.02-1.74)$ & 98 & 48.5 & 113 & 56.8 & 0.9 \\
\hline GG & 78 & 18.0 & 119 & 14.3 & 0.01 & $1.56(1.08-2.25)$ & 50 & 24.8 & 25 & 12.6 & 0.008 \\
\hline A & 487 & 56.2 & 1,027 & 61.7 & & & 206 & 51.0 & 235 & 59.0 & \\
\hline G & 379 & 43.8 & 637 & 38.3 & 0.008 & $1.25(1.06-1.49)$ & 198 & 49.0 & 163 & 41.0 & 0.02 \\
\hline \multicolumn{12}{|l|}{$\operatorname{rs} 2292239^{b}$} \\
\hline & 434 & & 846 & & & & 202 & & 200 & & \\
\hline $\mathrm{CC}$ & 127 & 29.3 & 304 & 35.9 & & 1 & 56 & 27.7 & 56 & 28.0 & 1 \\
\hline $\mathrm{CA}$ & 225 & 51.8 & 403 & 47.6 & 0.03 & $1.34(1.02-1.75)$ & 96 & 47.5 & 115 & 57.5 & 0.44 \\
\hline AA & 82 & 18.9 & 139 & 16.4 & 0.048 & $1.41(0.99-2.02)$ & 50 & 24.8 & 29 & 14.5 & 0.068 \\
\hline $\mathrm{C}$ & 479 & 55.2 & 1,011 & 59.8 & & & 208 & 51.5 & 227 & 56.8 & \\
\hline A & 389 & 44.8 & 681 & 40.2 & 0.03 & $1.21(1.02-1.43)$ & 196 & 48.5 & 173 & 43.2 & 0.1 \\
\hline \multicolumn{12}{|l|}{ rs $10876864^{c}$} \\
\hline & 427 & & 842 & & & & 201 & & 194 & & \\
\hline AA & 96 & 22.5 & 234 & 27.8 & & 1 & 38 & 19.0 & 47 & 24.2 & 1 \\
\hline $\mathrm{AG}$ & 218 & 51.2 & 408 & 48.5 & 0.07 & $1.30(0.97-1.76)$ & 101 & 50.5 & 102 & 52.6 & 0.29 \\
\hline GG & 112 & 26.3 & 200 & 23.8 & 0.06 & $1.36(0.97-1.93)$ & 61 & 30.5 & 45 & 23.2 & 0.07 \\
\hline A & 410 & 48.1 & 876 & 52.0 & & & 177 & 44.2 & 196 & 50.5 & \\
\hline G & 442 & 51.9 & 808 & 48.0 & 0.06 & $1.17(0.99-1.38)$ & 223 & 55.8 & 192 & 49.5 & 0.08 \\
\hline
\end{tabular}

Genotypic $p$ values in case-control and age-stratified analyses were calculated by fixing the common homozygote in controls as the standard (OR 1), and separately comparing heterozygotes and homozygotes for the minor allele with it. ORs for all allelic comparisons (including footnotes) are calculated for the minor allele (defined by the frequency in controls) vs the major allele

$p$ values in the right hand column show comparisons between patients with paediatric onset ( $\leq 16$ years) and adult onset ( $>16$ years)

Allelic frequencies were compared between paediatric and adult-onset patients to explore differences within patients (data in the table), and afterwards, they were separately compared with control allelic frequencies to identify the significantly different group (data in footnotes)

Analysis was performed using $2 \times 2$ contingency tables and $\chi^{2}$ test comparison in all cases

T1D, type 1 diabetes

${ }^{a}$ Minor allele frequency of T1D $\leq 16$ vs controls: OR 1.55 (95\% CI 1.24-1.94), $p=0.00008$; T1D $>16$ vs controls: OR 1.12 (95\% CI $\left.0.89-1.41\right), p=0.33$

${ }^{\mathrm{b}}$ Minor allele frequency of T1D $\leq 16$ vs controls: OR 1.40 (95\% CI 1.12-1.75), $p=0.002$; T1D > 16 vs controls: OR 1.13 (95\% CI $\left.0.90-1.42\right), p=0.27$

${ }^{\mathrm{c}}$ Minor allele frequency of T1D $\leq 16$ vs controls: OR 1.37 (95\% CI 1.09-1.71), $p=0.005$; T1D > 16 vs controls: OR 1.06 (95\% CI 0.85-1.33), $p=0.59$

Linkage disequilibrium between the three markers in our population was similar to that described for the HapMap CEU (Centre d'Etude du Polymorphisme [Utah residents with Northern and Western European ancestry]) cohort (ESM Fig. 1).

\section{Discussion}

The association between the region $12 \mathrm{q} 13$ and age at onset of type 1 diabetes was analysed by Hakonarson et al. in a mixed population of European and American participants with European ancestry [3]. They found a trend towards association in three SNPs, which was strongest in rs773107 $(p=0.047)$, and showed that homozygotes for the susceptibility genotype progressed to disease 1 year earlier (median $=8.0$ years; interquartile range 4.0-11.7) than homozygotes for the protective genotype (median $=9.0$ years; interquartile range 5.0-13.0). Although adult patients were included in the study, $75 \%$ had a diagnosis before age 13 years.

We selected rs2292239 as being the strongest signal originally identified in $12 \mathrm{q} 13$ and we had $86.5 \%$ statistical power to detect the previously described OR [2]. Out of the three SNPs studied by Hakonarson et al., we selected the marker most associated with age at disease onset (rs 773107, $r^{2}=0.81$ with $\left.\mathrm{rs} 2292239\right)$ and we had $73.25 \%$ statistical power [3]. However, according to the higher effect described for rs773107 in Wang et al. [5], our statistical power would be $97.52 \%$. We also selected $\operatorname{rs} 10876864\left(r^{2}=0.57\right.$ with rs2292239) to explore new variability in the region, having 
Table 2 Analysis of the effect of rs 773107 , rs 2292239 and rs10876864 in T1D age at onset considering the different genotypes, alleles and homozygotes of the risk alleles
Differences in age at onset between genotypes were calculated with the Kruskall-Wallis test. Differences in alleles and carriers were calculated using the Mann-Whitney $U$ test

\begin{tabular}{|c|c|c|c|c|}
\hline \multirow[t]{2}{*}{ Polymorphism } & \multirow[t]{2}{*}{$n$} & \multicolumn{2}{|c|}{ Age at onset (years) } & \multirow[t]{2}{*}{$p$ value } \\
\hline & & Mean (SD) & Median (1st and 3rd quartile) & \\
\hline \multicolumn{5}{|l|}{ rs773107 } \\
\hline AA & 115 & $19.5(9.9)$ & $18.0(12.0,26.0)$ & \\
\hline $\mathrm{AG}$ & 211 & $19.4(11.4)$ & $17.0(11.0,27.0)$ & 0.002 \\
\hline GG & 75 & $15.0(11.4)$ & $13.0(6.0,23.0)$ & \\
\hline A & 441 & $19.5(10.6)$ & $18.0(12.0,26.0)$ & \\
\hline G & 361 & $17.6(11.5)$ & $15.0(8.0,25.0)$ & 0.003 \\
\hline A carrier & 326 & $19.4(10.9)$ & $17.5(12.0,26.3)$ & \\
\hline GG genotype & 75 & $15.0(11.4)$ & $13.0(6.0,23.0)$ & 0.0004 \\
\hline \multicolumn{5}{|l|}{ rs2292239 } \\
\hline $\mathrm{CC}$ & 112 & $18.9(10.0)$ & $16.5(12.0,25.0)$ & \\
\hline $\mathrm{AC}$ & 211 & $19.9(11.4)$ & $18.0(12.0,28.0)$ & 0.001 \\
\hline AA & 79 & $14.8(10.8)$ & $13.0(6.0,23.0)$ & \\
\hline $\mathrm{C}$ & 435 & $19.4(10.7)$ & $17.0(12.0,26.0)$ & \\
\hline A & 369 & $17.7(11.4)$ & $15.0(9.0,25.0)$ & 0.013 \\
\hline $\mathrm{C}$ carrier & 323 & $19.6(11.0)$ & $18.0(12.0,27.0)$ & \\
\hline AA genotype & 79 & $14.8(10.8)$ & $13.0(6.0,23.0)$ & 0.0002 \\
\hline \multicolumn{5}{|l|}{ rs 10876864} \\
\hline GG & 106 & $16.7(10.4)$ & $15.0(8.8,24.0)$ & \\
\hline GA & 203 & $19.0(11.6)$ & $17.0(11.0,26.0)$ & 0.127 \\
\hline $\mathrm{AA}$ & 85 & $19.7(10.3)$ & $19.0(12.0,26.0)$ & \\
\hline G & 415 & $17.8(11.0)$ & $15.0(10.0,25.0)$ & \\
\hline $\mathrm{A}$ & 373 & $19.3(11.0)$ & $18.0(12.0,26.0)$ & 0.051 \\
\hline A carrier & 288 & $19.2(11.2)$ & $17.0(11.3,26.0)$ & \\
\hline GG genotype & 106 & $16.7(10.4)$ & $15.0(8.8,24.0)$ & 0.058 \\
\hline
\end{tabular}

$74.25 \%$ statistical power to detect its effect [3]. Statistical power was calculated for $\alpha=0.05$ in all cases.

In the present study, we aimed at replicating the age-at-onset analysis performed by Hakonarson et al. in a population with $50 \%$ paediatric and $50 \%$ adult-onset patients with ages at disease onset from 0 to 65 years. The age-at-onset study in a group with a wider age span allowed us to better discriminate the differences suggested by Hakonarson et al., and we found differences in disease onset of between 2 and 5 years in genotypes, alleles and homozygotes for the risk allele (Table 2), while Hakonarson et al. showed differences of 1 year between protective and risk genotypes. They did not provide analysis for alleles or homozygotes for the risk genotype.

Wang et al. [5] studied several 12q13 markers in a group with early-onset ( $\leq 17$ years, $62 \%$ of patients) and late-onset patients ( $>17$ years, 38\%), all North American individuals with European ancestry. The authors studied the SNPs in each group separately and found association between rs773107 and rs2292239 with both age groups and in the joint analysis. Concerning rs10876864, the association in paediatric and adult patients shows some differences that may point to an age-at-onset effect. The early-onset patients showed a stronger association (OR 1.3, $p=2 \times 10^{-5}$ ) than the adult-onset patients
(OR 1.2, $p=0.03$ ) and the heterogeneity analysis was borderline significant $(p=0.10)$, suggesting differences between both subsets. This opens the door to the possibility that the results in the late-onset group depend on the cut-off applied in stratified groups. An analysis considering age as a continuous variable, not shown in Wang's study, would help to shed light on this matter. Interestingly, among the other SNPs studied, the authors found one association in the earlyonset group (rs10521065, OR 1.3, $p=10^{-4}$ ) that was not statistically significant in late-onset patients (OR 1.1, $p=0.11$ ). This would suggest that the region could have an effect on age-at-onset, as Hakonarson et al. pointed out. Wang et al. did not provide a detailed description of their sample (maximum, minimum, mean and median age at onset), which hampers further comparison between their patients and ours.

There are more than ten genes in 12q13 and at least three of them (ERBB3, RPS26 and IKZF4) are good candidates for type 1 diabetes pathogenesis. The polymorphisms described in $12 q 13$ could putatively mark more than one causal gene, and it is possible that only one of them affects the age at onset. This, together with different linkage patterns that would modify the linkage disequilibrium between marker SNPs and aetiological variants through different populations, 
could explain the differences seen between both our study and Hakonarson's [3], and the latter work by Wang et al. [5].

Acknowledgements We thank C. Martínez and M A. García-Martínez for expert technical assistance. E. Urcelay works for the Fundación para la Investigación Biomédica Hospital Clínico San Carlos.

Duality of interest The authors declare that there is no duality of interest associated with this manuscript.

\section{References}

1. The Wellcome Trust Case-Control Consortium (2007) Genomewide association study of 14,000 cases of seven common diseases and 3,000 shared controls. Nature 447:661-678
2. Todd JA, Walker NM, Cooper JD et al (2007) Robust associations of four new chromosome regions from genome-wide analyses of type 1 diabetes. Nat Genet 39:857-864

3. Hakonarson H, Qu HQ, Bradfield JP et al (2008) A novel susceptibility locus for type 1 diabetes on Chr12q13 identified by a genome-wide association study. Diabetes 57:1143-1146

4. Hakonarson H, Grant SF, Bradfield JP et al (2007) A genome-wide association study identifies KIAA0350 as a type 1 diabetes gene. Nature 448:591-594

5. Wang H, Jin Y, Reddy MV et al (2010) Genetically dependent ERBB3 expression modulates antigen presenting cell function and type 1 diabetes risk. PLoS One 5:e11789

6. Dubois PC, Trynka G, Franke L et al (2010) Multiple common variants for celiac disease influencing immune gene expression. Nat Genet 42:295-302

7. Ferreira RC, Pan-Hammarstrom Q, Graham RR et al (2010) Association of IFIH1 and other autoimmunity risk alleles with selective IgA deficiency. Nat Genet 42:777-780 